\title{
Serum level of octanoic acid predicts the efficacy of chemotherapy for colorectal cancer
}

\author{
TAKAO IEMOTO $^{1}$, SHIN NISHIUMI $^{1}$, TAKASHI KOBAYASHI ${ }^{1}$, SEIJI FUJIGAKI $^{1}$, \\ TETSUYA HAMAGUCHI ${ }^{2}$, KEN KATO $^{2}$, HIROKAZU SHOJI ${ }^{2}$, YASUHIRO MATSUMURA ${ }^{3}$, \\ KAZUFUMI HONDA ${ }^{4}$ and MASARU YOSHIDA M, $^{1,5}$ \\ ${ }^{1}$ Division of Gastroenterology, Department of Internal Medicine, Kobe University Graduate School of Medicine, \\ Kobe, Hyogo 650-0017; ${ }^{2}$ Division of Gastrointestinal Medical Oncology, National Cancer Center Hospital, \\ Tokyo 104-0045; ${ }^{3}$ Division of Developmental Therapeutics, Exploratory Oncology Research and Clinical Trial Center, \\ National Cancer Center, Kashiwa, Chiba 277-8577; ${ }^{4}$ Division of Chemotherapy and Clinical Research, \\ National Cancer Center Research Institute, Tokyo 104-0045; ${ }^{5}$ Division of Metabolomics Research, \\ Department of Internal Related, Kobe University Graduate School of Medicine, Kobe, Hyogo 650-0017; \\ ${ }^{6}$ AMED-CREST, AMED, Kobe, Hyogo 650-0017, Japan
}

Received May 11, 2018; Accepted September 19, 2018

DOI: $10.3892 / 01.2018 .9731$

\begin{abstract}
The survival times of patients with advanced colorectal cancer (CRC) have increased due to the introduction of chemotherapy involving irinotecan and cetuximab. However, further studies are required on the effective pretreatment methods for identifying patients with CRC who would respond to particular treatments. The aim of the present study was to identify biomarkers for predicting the efficacy of chemotherapy for CRC. A total of 123 serum samples were collected from 31 patients with CRC just prior to each of the first four rounds of chemotherapy. Serum metabolome analysis was performed using a multiplatform metabolomics system, and univariate Cox regression hazards analysis of the time to disease progression was conducted. Octanoic acid and 1,5-anhydro-D-glucitol were identified as biomarker candidates. In addition, the serum level of octanoic acid was indicated to be significantly associated with the time to disease progression (hazard ratio, 3.3; 95\% confidence interval, 1.099-11.840; $\mathrm{P}=0.033$ ). The serum levels of fatty acids, in particular polyunsaturated fatty acids, tended to be downregulated in the partial response group. The findings of the present study suggest that the serum level of octanoic acid may serve as a useful predictor for the prognosis of CRC.
\end{abstract}

Correspondence to: Dr Masaru Yoshida, Division of Gastroenterology, Department of Internal Medicine, Kobe University Graduate School of Medicine, 7-5-1 Kusunoki-cho, Chuo, Kobe, Hyogo 650-0017, Japan

E-mail: myoshida@med.kobe-u.ac.jp

Key words: treatment efficacy, colorectal cancer, predictive biomarker, gas chromatography/mass spectrometry, liquid chromatography/mass spectrometry

\section{Introduction}

In Japan, the mortality rate of patients with colorectal cancer (CRC) has been increasing. According to the vital statistics of Japan, in 2015, CRC was the leading cause of cancer-associated mortality in females and the third leading cause of cancer-associated mortality in males (1). The 5-year survival rate of patients with CRC following curative resection is $\sim 80 \%$, but $10-20 \%$ of cases are unresectable (2). In the last two decades, the mean survival time of patients with advanced CRC has increased from 1 year to $\sim 2$ years due to the introduction of chemotherapy drugs, including irinotecan and oxaliplatin. These therapeutic agents are used in combination with fluorouracil and leucovorin as standard regimens, FOLFILI and FOLFOX4, for advanced CRC (3-5). Recently, targeted biological therapeutic agents have proved to be useful for treating CRC (6,7). Cetuximab, a targeted biological therapeutic agent, has significantly improved the survival times of patients with CRC with metastatic lesions, particularly when the cancer is refractory to the aforementioned anticancer drugs (8). According to the BOND-1 study (9), combination therapy with cetuximab and irinotecan produced a higher response rate compared with cetuximab alone. In addition, cetuximab has the capacity to reverse drug resistance by abrogating drug efflux, restoring apoptosis and impairing DNA repair activity when administered in combination with irinotecan $(8,9)$.

A number of randomized trials have suggested that mutations in the Kirsten rat sarcoma viral oncogene homolog (KRAS) and v-Raf murine sarcoma viral oncogene homolog B (BRAF) genes are negative prognostic predictors among patients with metastatic CRC who are treated with anti-epidermal growth factor receptor (EGFR) antibodies (10-13). The efficacy of KRAS mutations as predictive biomarkers is well established, but the effects of BRAF mutations remain unknown (14). Further investigation is required on the effective pretreatment 
methods for identifying patients with CRC that would respond to particular treatments. The ability to predict the efficacy of particular treatments in each patient during the pretreatment period would allow doctors to provide a customized treatment for each patient, which may improve the quality of life and prognosis of the patient. Therefore, it is necessary to identify biomarkers that could be used to predict the efficacy of treatments for CRC.

Metabolomics is the comprehensive study of low molecular weight molecules, known as metabolites, in a particular organism or tissue. Metabolomics is a useful approach to understanding the state of a patient's body, as metabolites and their concentrations directly reflect the underlying biochemical activity and state of cells/tissues. In addition, metabolites represent the endpoint of the omics cascade and therefore can be directly linked to molecular phenotypes (15). Therefore, metabolomics is employed as a tool for discovering candidate biomarkers that may aid disease diagnosis, toxicological testing, prognosis and risk assessment (16-18). We have previously demonstrated the usefulness of metabolomics for facilitating the diagnosis and evaluation of pancreatic cancer and CRC (19-22). However, to the best of our knowledge, a limited number of studies have investigated whether metabolite biomarkers could be used to predict the response to cancer treatment. A previous study involving high-resolution magic angle spinning magnetic resonance spectroscopy indicated that a high concentration of glycine was correlated with poor progression-free survival time in patients with locally advanced rectal cancer (23). The aim of the present study was to use gas chromatography/mass spectrometry (GC/MS) and liquid chromatography/MS (LC/MS) to identify biomarkers that could be used to predict the response to chemotherapy (cetuximab plus irinotecan or cetuximab alone) for CRC. First, biomarker candidates were searched for among serum metabolites by performing a semi-quantitative analysis without the corresponding stable isotopes. A quantitative analysis was subsequently conducted using the corresponding stable isotopes for the selected biomarker candidates and their efficacy was validated.

\section{Materials and methods}

Patients and treatment. The present study was conducted as part of a phase II trial of chemotherapy for CRC, a clinical trial of third-line or later treatment for CRC (24), involving cetuximab plus irinotecan or cetuximab alone, performed at the National Cancer Center Hospital (Tokyo, Japan). The present study was approved by the Ethics Committees of the National Cancer Center Hospital and Kobe University Graduate School of Medicine (Kobe, Japan). Written informed consent was obtained from all patients prior to participation in the present study. The patients were recruited from the National Cancer Center Hospital between December 2008 and March 2015. The inclusion criteria were as follows: A histologically confirmed diagnosis of metastatic CRC that was surgically unresectable or had recurred; having received ineffective irinotecan, oxaliplatin or fluoropyrimidine-based chemotherapy; an age of $>20$ years; an Eastern Cooperative Oncology Group performance status score of 0-2 (25); a life expectancy of at least 2 months; possessing the wild-type
KRAS gene; and having at least one radiologically measurable lesion according to the Response Evaluation Criteria in Solid Tumors (RECIST) version 1.0 (26). The present study, as aforementioned, was conducted as an accompaniment to a phase II clinical trial of third-line or later treatment for $\mathrm{CRC}$, and in the event that irinotecan alone was not able to produce sufficient therapeutic effects, cetuximab + irinotecan or cetuximab alone was expected to be effective as a third-line treatment. Therefore, 'having received ineffective irinotecan' was included in the inclusion criteria. In addition, the minimum treatment-free period between the end of the previous therapy and the day of registration was 4, 2 and 4 weeks for patients who had received prior chemotherapy, radiotherapy and major surgery, respectively. The exclusion criteria were as follows: Having received EGFR signal transduction inhibitors or anti-EGFR antibodies; having suffered double cancer within the past 5 years; and were suffering from active inflammation or severe complications, including heart disease, diabetes, and ileus or interstitial lung disease.

A total of 31 patients with CRC, including 21 males and 10 females, participated in the present study (median age, 65; range, 48-79 years). The subjects were allocated to groups A and B. Patients who satisfy the following conditions are regarded as 'Irinotecan unreliable' and were allocated in group B; having advanced peritoneal metastasis, having ascites in the pelvic cavity and upper abdomen within 14 days prior to registration, drainage of ascites is done within 14 days prior to registration. However, patients who did not satisfy the aforementioned conditions were allocated in group A. The patients in group A $(n=27)$, consisting of 19 males and 8 females, were treated with cetuximab and irinotecan, and the patients in group B $(n=4)$, consisting of 2 males and 2 females, were treated with cetuximab alone. The patients received cetuximab on day 1 of a 7-day treatment cycle. Cetuximab was initially administered at a dose of $400 \mathrm{mg} / \mathrm{m}^{2}$ as a 2-h infusion and was subsequently administered at a weekly dose of $250 \mathrm{mg} / \mathrm{m}^{2}$ as a 1-h infusion. Irinotecan was administered once every 2 weeks, starting on day 1 , at a dose of $150 \mathrm{mg} / \mathrm{m}^{2}$ as a 90 min infusion. Irinotecan administered consecutively with cetuximab (Fig. 1). The aforementioned treatments were continued until disease progression or unacceptable toxicities occurred. Responses were evaluated using computed tomography (CT) scans obtained every 8 weeks for each patient and measuring the lesions using the RECIST version 1.0 criteria.

Serum samples. Serum samples were collected from all patients prior to each of the first four rounds of chemotherapy (days 1,8, 15 and 22) at the National Cancer Center Hospital (Fig. 1) and were used for the metabolome analysis. At $30 \mathrm{~min}$ post-blood sampling, the samples were centrifuged at 1,500 $\mathrm{x} g$ for $10 \mathrm{~min}$ at $4^{\circ} \mathrm{C}$. Next, the obtained serum was immediately transferred to a clean tube and stored at $-80^{\circ} \mathrm{C}$ until it was used. The serum samples were subjected to analysis by GC/MS and LC/MS.

Chemicals and isotopes. $\left[\mathrm{U}^{13} \mathrm{C}_{6}\right.$ (98\%)]-labeled 1,5-Anhydro-D-glucitol, ([U- ${ }^{13} \mathrm{C}_{6}$ (98\%)]-labeled 1,5-AG) and $\left[1-{ }^{13} \mathrm{C}\right]$ - labeled Octanoic acid were obtained from Cambridge Isotope Laboratories, Inc. (Tewksbury, MA, USA). Octanoic acid- $1-{ }^{13} \mathrm{C}, 2$-isopropylmalic acid (as an internal 


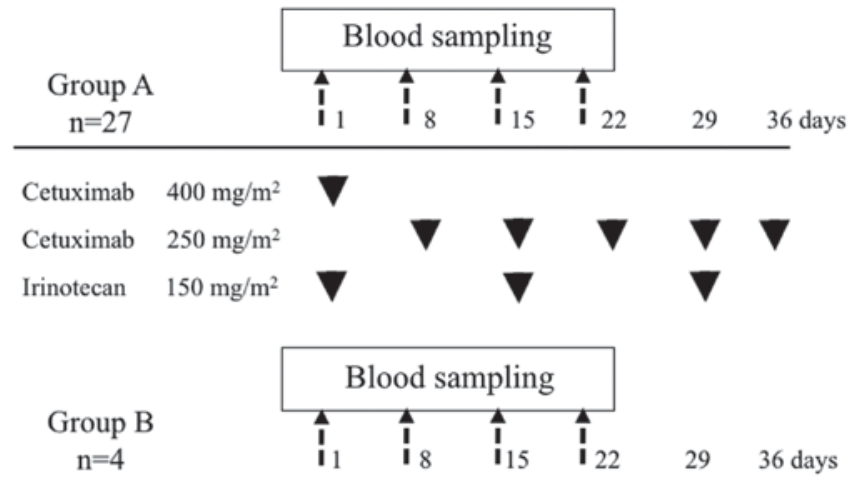

Cetuximab $\quad 400 \mathrm{mg} / \mathrm{m}^{2}$

Cetuximab $250 \mathrm{mg} / \mathrm{m}^{2}$

Figure 1. Chemotherapy protocols. The patients were allocated to group A or group B. The patients in group $A(n=27)$ were treated with cetuximab and irinotecan. The patients in group $B(n=4)$ were treated with cetuximab alone.

standard), 2-bromohypoxanthine (as an internal standard) and 10-camphorsulfonic acid (as an internal standard) were purchased from Sigma-Aldrich; Merck KGaA (Darmstadt, Germany). 1,5-AG and octanoic acid were acquired from Wako Pure Chemical Industries, Ltd. (Osaka, Japan). Dilauroylphosphatidylcholine (PC12:0/12:0) (as an internal standard) was obtained from Avanti Polar Lipids, Inc. (Alabaster, AL, USA).

GC/MS procedures. The GC/MS extraction and derivatization procedures were performed according to the methods described in our previous studies $(20-22,27)$. A total of $20 \mu 1$ of each sample was dispensed into a 1.5-ml Eppendorf tube. The samples were then extracted with $250 \mu \mathrm{l}$ methanol with or without the corresponding stable isotopes, prior to being shaken in a vortex mixer. A total of $10 \mu 12$-isopropylmalic acid $(0.5 \mathrm{mg} / \mathrm{ml})$ was added as an internal standard. The mixture was incubated at $1,200 \mathrm{rpm}$ for $30 \mathrm{~min}$ at $37^{\circ} \mathrm{C}$. The mixture was centrifuged at $19,300 \times \mathrm{g}$ for $5 \mathrm{~min}$ at $4^{\circ} \mathrm{C}$ and $200 \mu \mathrm{l}$ supernatant was transferred to a new Eppendorf tube capped with a pierced cap. Subsequent to being centrifuged for $30 \mathrm{~min}$ in a vacuum concentrator (Thermo SpeedVac; Thermo Fisher Scientific, Inc., Waltham, MA, USA), the mixture was freeze-dried overnight. For the derivatization, $80 \mu 1$ methoxamine hydrochloride in pyrimidine $(20 \mathrm{mg} / \mathrm{ml})$ were added as the first derivatizing agent. The mixture was subsequently incubated at $1,200 \mathrm{rpm}$ for $90 \mathrm{~min}$ at $30^{\circ} \mathrm{C}$. The second derivatizing agent, $40 \mu \mathrm{l} \mathrm{N}$-methyl-N-(trimethylsilyl) trifluoroacetamide, was added and the mixture was incubated at $1,200 \mathrm{rpm}$ for $30 \mathrm{~min}$ at $37^{\circ} \mathrm{C}$. The mixture was centrifuged at $19,300 \mathrm{x} \mathrm{g}$ for $5 \mathrm{~min}$ at $25^{\circ} \mathrm{C}$, and the supernatant was transferred to a vial for analysis by GC/MS. The GC/MS analysis was performed on a GCMS-TQ8040 GC-MS/MS system (Shimadzu Corporation, Kyoto, Japan) with a fused silica capillary column (BPX5; inner diameter: $30 \mathrm{~m}$ x $0.25 \mathrm{~mm}$; film thickness, $0.25 \mu \mathrm{m}$; SGE Analytical Science, Melbourne, Australia). The data processing was performed according to previously described methods $(21,22,27)$. The MS data were exported to a personal computer, on which the GCMS solution software ver. 4.30 (Shimadzu Corporation) had been installed, and the peaks of the targeted metabolites and the corresponding stable isotopes were detected by the software and subsequently checked manually. According to the methods described in our previous study (22), quality control plasma samples were prepared to ensure the reproducibility of the acquired metabolomic data. In the analysis of the quality control plasma samples, coefficients of variation (CV) for the ratio of the peak area value for each metabolite to that for the internal standard were calculated, following the exclusion of metabolites with a $\mathrm{CV}>30 \%$. In the semi-quantitative analysis, the peak intensity of each metabolite was normalized to that of the internal standard. In the GC/MS-based quantitative analysis of the targeted metabolites (octanoic acid and 1,5-AG), the peak area of each metabolite was corrected using the data for the corresponding stable isotope ([U- $\left.{ }^{13} \mathrm{C}_{6}(98 \%)\right]$-labeled 1,5-AG and $\left[1-{ }^{13} \mathrm{C}\right]$-labeled Octanoic acid), and quantitation was conducted based on multi-point calibration curves.

LC/MS procedures. For the LC/MS-based hydrophilic metabolite analysis, $20 \mu \mathrm{l}$ of each sample was mixed with $900 \mu \mathrm{l}$ of a solvent mixture $\left(\mathrm{H}_{2} \mathrm{O}: \mathrm{CHCl}_{3}\right.$ :methanol, 1:1:2.5) containing $1 \mathrm{mM}$ 2-bromohypoxanthine and $1 \mathrm{mM}$ 10-camphorsulfonic acid as internal standards. The mixture was agitated at $1,400 \mathrm{rpm}$ for $30 \mathrm{~min}$ at $37^{\circ} \mathrm{C}$. Subsequently, $630 \mu \mathrm{l}$ of the supernatant was transferred to a new Eppendorf tube, and $280 \mu \mathrm{l}$ distilled water was added to the tube. Subsequent to being mixed, the mixture was centrifuged at $16,000 \mathrm{x} \mathrm{g}$ for $5 \mathrm{~min}$ at $4^{\circ} \mathrm{C}$, and $500 \mu \mathrm{l}$ of the resultant supernatant was passed through an ultrafiltration filter, prior to being centrifuged at $14,000 \mathrm{x}$ g for $60 \mathrm{~min}$ at $4^{\circ} \mathrm{C}$. The collected solution was dried via centrifugal concentration and lyophilization, and reconstituted with $100 \mu 1$ water. The resultant supernatant was subjected to analysis. For the LC/MS-based lipid analysis, $10 \mu \mathrm{l}$ of each sample was mixed with $80 \mu \mathrm{l}$ methanol and $10 \mu 1500 \mathrm{ppb} \mathrm{PC12:0/12:0} \mathrm{dissolved}$ in methanol as an internal standard. The mixture was put on ice for $10 \mathrm{~min}$. Following centrifugation at $16,000 \mathrm{x} \mathrm{g}$ for $5 \mathrm{~min}$ at $4^{\circ} \mathrm{C}, 60 \mu \mathrm{l}$ of the supernatant was transferred to a new Eppendorf tube and subjected to analysis.

According to the method described in our previous studies $(20,27)$, the LC/MS analysis was performed using a Nexera LC system (Shimadzu Corporation) equipped with two LC-30AD autosamplers, a CTO-20AC column oven and a CBM-20A control module, coupled with an LCMS-8040 triple quadruple mass spectrometer (Shimadzu Corporation). The analytical conditions of LC/MS were as follows: Electrospray voltage: $3.5 \mathrm{kV},-3.5 \mathrm{kV}$; Curve desolvation line (CDL): $250^{\circ} \mathrm{C}$; Heat block temperature: $400^{\circ} \mathrm{C}$; Nebulizing gas $\left(\mathrm{N}_{2}\right): 3.01 / \mathrm{min}$; Drying gas pressure: $15 \mathrm{l} / \mathrm{min}$; CID gas (Argon) pressure: $0.23 \mathrm{MPa}$. The cationic hydrophilic metabolites were separated using a pentafluorophenyl column (Discovery HS F5; 150x2.1 $\mu \mathrm{m} ; 3 \mu \mathrm{m}$; Supelco, Inc., Bellefonte, PA, USA) with a guard column $(20 \times 2.1 \mu \mathrm{m} ; 3 \mu \mathrm{m}$; GL Sciences, Inc., Tokyo, Japan), while the anionic hydrophilic metabolites were separated using an octadecylsilylated silica column (InertSustain C18; 150x2.1 $\mu \mathrm{m} ; 3 \mu \mathrm{m}$; GL Sciences, Inc., Tokyo, Japan). The mobile phase used for the analysis of the cationic metabolites was composed of A: $0.1 \%$ formic acid in water and B: $100 \%$ Acetonitrile. The flow rate was $0.3 \mathrm{ml} / \mathrm{min}$, and the column oven temperature was set at $40^{\circ} \mathrm{C}$. The gradient 
Table I. Patient characteristics and evaluation of the response to chemotherapy.

\begin{tabular}{|c|c|c|c|}
\hline Variables & PR & Non-PR & P-value \\
\hline Total, $\mathrm{n}$ & 7 & 24 & \\
\hline Sex, $n$ & & & $0.784^{\mathrm{a}}$ \\
\hline Male & 5 & 16 & \\
\hline Female & 2 & 8 & \\
\hline Median age (range), years & $67.0(48-77)$ & $64.5(51-79)$ & $0.607^{\mathrm{b}}$ \\
\hline PS, n & & & $0.664^{\mathrm{a}}$ \\
\hline 0 & 2 & 9 & \\
\hline 1 & 5 & 15 & \\
\hline BMI & 21.2 & 21.6 & $0.499^{\mathrm{b}}$ \\
\hline Group, $\mathrm{n}^{\mathrm{c}}$ & & & $0.247^{\mathrm{a}}$ \\
\hline A & 7 & 20 & \\
\hline B & 0 & 4 & \\
\hline OS, days & 433 & 336 & $0.028^{\mathrm{d}}$ \\
\hline PFS, days & 253 & 165.5 & $0.043^{\mathrm{d}}$ \\
\hline TTP, days & 162 & 102.5 & $0.047^{\mathrm{d}}$ \\
\hline
\end{tabular}

${ }^{a} \mathrm{P}$-values were calculated using Pearson's $\chi^{2}$ test. ${ }^{\mathrm{b}} \mathrm{P}$-values were calculated using the Student's t-test. 'Group A, cetuximab plus irinotecan; Group B, cetuximab alone. ${ }^{\mathrm{P}} \mathrm{P}$-values were calculated using the log-rank test. PR, partial response; Non-PR, non-partial response; OS, overall survival; PFS, progression-free survival; TTP, time to progression; BMI, body mass index; PS, performance status score.

program for mobile phase $\mathrm{B}$ was as follows: $0 \mathrm{~min}, 0 \%$; $7 \mathrm{~min}, 0 \%$; $20 \mathrm{~min}, 40 \%$; $20.1 \mathrm{~min}, 100 \%$; $25 \mathrm{~min}, 100 \%$; $25.1 \mathrm{~min}, 0 \%$; and $35 \mathrm{~min}, 0 \%$. The mobile phase used for the analysis of the anionic metabolites was composed of A: Water containing $15 \mathrm{mM}$ acetic acid and $10 \mathrm{mM}$ tributylamine, and B: $100 \%$ Methanol. The flow rate was $0.3 \mathrm{ml} / \mathrm{min}$, and the column oven temperature was set at $35^{\circ} \mathrm{C}$. The gradient program for mobile phase B was as follows: $0 \mathrm{~min}, 0 \%$; $0.5 \mathrm{~min}, 0 \%$; $20 \mathrm{~min}, 75 \%$; $20.1 \mathrm{~min}, 98 \%$; $24 \mathrm{~min}, 98 \%$; $24.1 \mathrm{~min}, 0 \%$; and $30 \mathrm{~min}, 0 \%$ (28). The $\mathrm{m} / \mathrm{z}$ value and retention time for each metabolite are listed in our previous studies (28). Lipids were separated using an octadecylsilylated silica column (InertSustain C18, 100x2.1 $\mu \mathrm{m} ; 3 \mu \mathrm{m}$; GL Sciences, Inc.) with a guard column (10x3 $\mu \mathrm{m} ; 5 \mu \mathrm{m}$; GL Sciences). The mobile phase used for the lipid analysis consisted of A: $20 \mathrm{mM}$ ammonium acetate in water and B: $100 \%$ Methanol. The flow rate was $0.4 \mathrm{ml} / \mathrm{min}$, and the column oven temperature was set at $40^{\circ} \mathrm{C}$. The gradient program for mobile phase B was as follows: $0 \mathrm{~min}, 80 \%$; $13 \mathrm{~min}, 98 \%$; $30 \mathrm{~min}, 98 \%$; $30.1 \mathrm{~min}, 80 \%$; and $35 \mathrm{~min}, 80 \%$. The aforementioned metabolites were mainly analyzed based on their physicochemical properties and/or spectral similarity with the molecules included in public/commercial spectral libraries (putative annotation), which are shown in our previous studies (28), as chemical reference standards could not be obtained.

Statistical analysis. Data are presented as mean \pm standard deviation. Univariate analyses were performed by conducting comparisons of treatment efficacy using the Mann-Whitney U test. Pearson's $\chi^{2}$ test was used to evaluate the differences between groups for categorized variables. The unpaired Student's t-test was used to measure the means and relationships of continuous variables. Receiver operating characteristic (ROC) curve analysis was used to evaluate the diagnostic performance of the targeted metabolites based on their area under the curve (AUC), sensitivity and specificity values. The optimal cut-off values for the selected serum metabolites were determined from their ROC curves. The Youden index was used to determine the optimal cut-off values for each serum metabolite (29). In univariate analysis of the selected serum metabolites, the likelihood ratio test was used to evaluate P-values. The time to progression (TTP) was analyzed using Cox proportional hazards regression analysis and the Kaplan-Meier method. TTP estimates were compared using the log-rank test. The patients who were lost to follow-up were evaluated using the data at the date of the last follow-up. $\mathrm{P}<0.05$ was considered to indicate a statistically significant difference. Kaplan-Meier curves were produced using the package 'survival' for $\mathrm{R}$. The log-rank test was performed using the statistical software R ver. 1.37 (http://www.jichi. ac.jp/saitama-sct/SaitamaHP.files/statmedOSX.html) (27,30). The other analyses were performed using the default conditions of JMP 11 (SAS Institute, Inc., Cary, NC, USA).

\section{Results}

Patient characteristics and serum samples. A total of 31 patients with CRC participated in the present study, and all were treated with chemotherapy, namely cetuximab plus irinotecan or cetuximab alone. The median duration of the follow-up period was 781.5 days, and the median survival time was 390 days. The median TTP was 152 days. In total, 123 serum samples were collected from the patients prior to the first to fourth rounds of treatment (days 1, 8, 15 and 22) 
Table II. Quantitative analysis of the targeted metabolites using gas chromatography/mass spectrometry.

\begin{tabular}{lccccccc}
\hline & \multicolumn{2}{c}{$\mathrm{PR}(\mathrm{n}=7)$} & & \multicolumn{2}{c}{ Non-PR $(\mathrm{n}=24)$} & & \\
\cline { 2 - 3 } Metabolite & Mean, $\mu \mathrm{M}$ & $\mathrm{SD}$ & & Mean, $\mu \mathrm{M}$ & $\mathrm{SD}$ & Fold-change $^{\mathrm{a}}$ & P-value $^{\mathrm{b}}$ \\
\hline Octanoic acid & 4.09 & 0.82 & & 5.06 & 1.07 & 0.81 & 0.043 \\
$1,5-\mathrm{AG}$ & 155.78 & 48.42 & & 106.78 & 34.25 & 1.46 & 0.026 \\
\hline
\end{tabular}

${ }^{a}$ The fold-change values were calculated based on the ratio of PR to non-PR. ${ }^{\mathrm{b}}$ The P-values were calculated using the Mann-Whitney U-test. PR, partial response; SD, standard deviation; 1,5-AG, 1,5-anhydro-D-glucitol.

(Fig. 1). One patient succumbed prior to providing a fourth sample.

Evaluation of treatment responses GC/MS. The therapeutic responses of the subjects are summarized in Table I. The overall survival period, progression-free survival and time to progression differed significantly between the partial response $(\mathrm{PR})$ and non-partial response (non-PR) groups $(\mathrm{P}=0.028$, $\mathrm{P}=0.043$ and $\mathrm{P}=0.047$, respectively), whereas there were no significant differences observed with regard to gender, age and body mass index. None of the patients achieved a complete response. A total of 7 patients (22.6\%) achieved a PR, while 14 patients $(45.2 \%)$ experienced stable disease and 9 patients $(29.0 \%)$ experienced progressive disease. Only 1 patient (3.2\%) could not be evaluated due to mortality prior to the first CT scan. The aim of the present study was to compare the serum levels of metabolites between the PR and non-PR groups. Therefore, the patient that could not be evaluated was included in the non-PR group, and overall, a total of 24 patients (77.4\%) were classed as non-PR.

In the GC/MS-based semi-quantitative analysis, a total of 111 metabolites were detected in the serum samples. The serum levels of each metabolite were compared between the PR and non-PR groups using the Mann-Whitney U test to determine their associations with the therapeutic response. The serum levels of two metabolites, including octanoic acid and 1,5-AG, exhibited significant intergroup differences [octanoic acid; $\mathrm{P}=0.030,1,5-\mathrm{AG} ; \mathrm{P}=0.006$, data not shown] prior to the first round of treatment.

To obtain quantitative data, a targeted metabolome analysis was performed using the corresponding stable isotopes. Stable isotope labeling can be used to achieve a more accurate quantification (31). The serum levels of octanoic acid and 1,5-AG, which were analyzed, along with their respective results are indicated in Table II. Box plots of the serum levels of these two metabolites in the PR and non-PR groups are presented in Fig. 2. In the quantitative analysis, it was indicated that the PR group exhibited significantly lower serum levels of octanoic acid compared with the non-PR group $(\mathrm{P}=0.043)$. By contrast, the serum level of 1,5-AG was significantly higher in the PR group compared with the non-PR group ( $\mathrm{P}=0.026$; Table II).

To evaluate the predictive accuracy of serum metabolite levels for predicting the efficacy of treatment for CRC, the AUC, sensitivity and specificity values were calculated for octanoic acid and 1,5-AG via ROC analysis. The optimal cut-off values for each serum metabolite were determined using the Youden index. The sensitivity, specificity and AUC
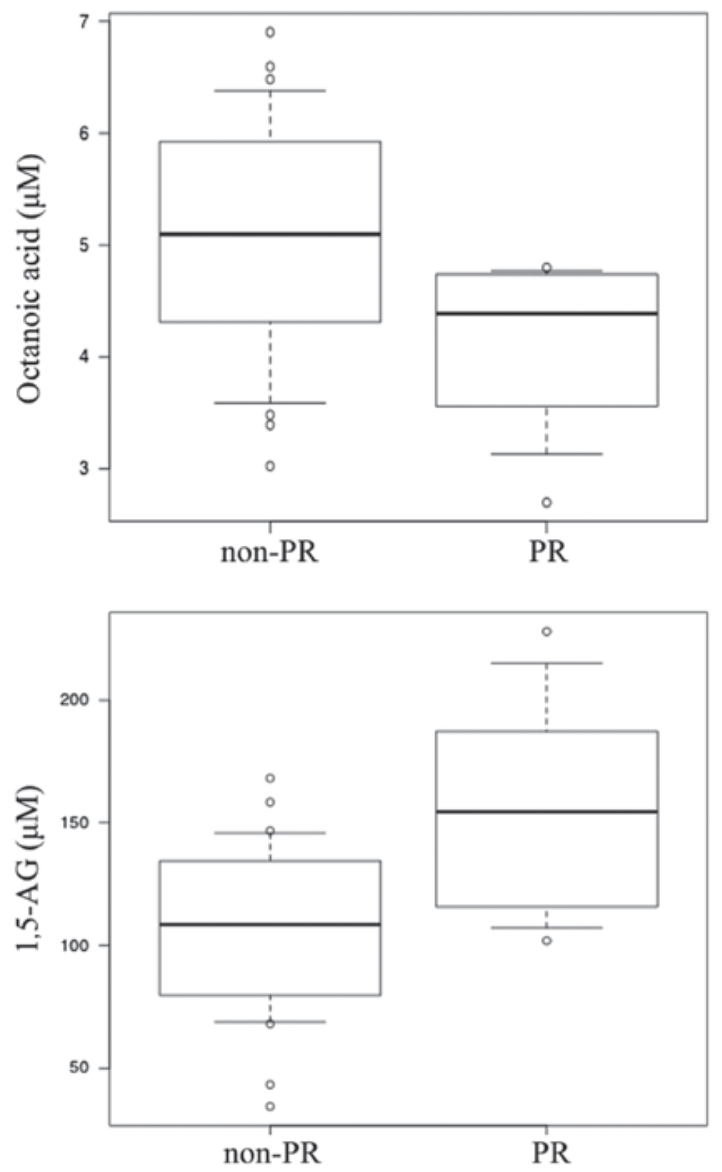

Figure 2. Box plots of the targeted metabolites. The box plots indicate the serum levels of the targeted metabolites, octanoic acid and 1,5-AG in the PR and non-PR groups. The boxes represent interquartile ranges. The horizontal bar within each box indicates the median value. The upper horizontal line of each box indicates the 75 th percentile, and the lower horizontal line indicates the 25th percentile. The upper horizontal bar above the box indicates the 90th percentile, and the horizontal bar below the box indicates the 10th percentile. The circles represent data points beyond the 10th and 90th percentiles. 1,5-AG, 1,5-anhydro-D-glucitol; PR, partial response.

of octanoic acid were $100 \%, 62.5 \%$, and $0.77 \mu \mathrm{M}$, respectively. The sensitivity, specificity and AUC of $1,5-\mathrm{AG}$ were $57.1 \%$, $91.7 \%$, and $0.78 \mu \mathrm{M}$, respectively. Therefore, octanoic acid and 1,5-AG exhibited moderate predictive accuracy (Table III). TTP was subsequently analyzed to evaluate the prognosis of the subjects. Disease progression was observed in 14 of the 31 patients during the observation period, and the median TTP was 162 days in the PR group and 102.5 days in the non-PR group (Table I). 
Table III. Predictive accuracy of the targeted metabolites.

\begin{tabular}{lcccc}
\hline Metabolite & AUC $(95 \% \mathrm{CI})$ & Sensitivity, $\%$ & Specificity, $\%$ & Cut-off value, $\mu \mathrm{M}^{\mathrm{a}}$ \\
\hline Octanoic acid & $0.77(0.59-0.95)$ & 100.0 & 62.5 & 4.8 \\
$1,5-\mathrm{AG}$ & $0.78(0.57-0.99)$ & 57.1 & 91.7 & 154.5 \\
\hline
\end{tabular}

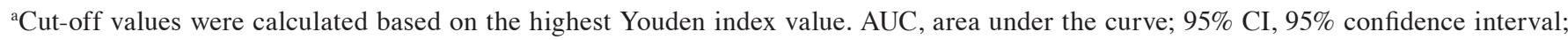
1,5-AG, 1,5-anhydro-D-glucitol.

Table IV. Cox proportional hazards regression analysis of the associations between serum metabolite concentrations and the time to progression.

\begin{tabular}{lcccr}
\hline & & \multicolumn{3}{c}{ Univariate analysis } \\
\cline { 3 - 5 } Metabolite & Cut-off value,$\mu \mathrm{M}$ & Hazard ratio & $95 \% \mathrm{CI}$ & P-value $^{\text {a }}$ \\
\hline Octanoic acid & $\leq 4.80 />4.80$ & 3.3 & $1.10-11.84$ & 0.0325 \\
$1,5-\mathrm{AG}$ & $\leq 154.5 />154.5$ & 1.1 & $0.35-4.92$ & 0.869 \\
\hline
\end{tabular}

aP-values were calculated using the likelihood ratio test. 95\% CI, 95\% confidence interval; 1,5-AG, 1,5-anhydro-D-glucitol.

Cox proportional hazards regression analysis was performed using the serum levels of octanoic acid and 1,5-AG, whose optimal cut-off values were determined from their ROC curves. Only the serum level of octanoic acid was indicated to be significantly associated with the TTP in the univariate analyses $[\leq 4.80 />4.80 \mu \mathrm{M}$; hazard ratio, 3.3 ; $95 \%$ confidence interval (CI), 1.1-12; $\mathrm{P}=0.033$; Table IV). Kaplan-Meier analysis indicated that the patients with lower serum octanoic acid levels achieved better prognoses compared with those with higher serum octanoic acid levels (Fig. 3). By contrast, the serum level of 1,5-AG was not indicated to be significantly associated with the TTP in the univariate analyses ( $\leq 154.5 />154.5 \mu \mathrm{M}$; hazard ratio, 1.1; 95\% CI, 0.35-4.92; $\mathrm{P}=0.87$; Table IV).

Furthermore, the changes in the levels of the targeted metabolites in the serum samples that were obtained from the first to the fourth round of treatment are indicated in Table V. The concentration of octanoic acid remained almost the same from prior to the first round of treatment to the fourth round of treatment in the PR and non-PR groups. By contrast, the concentration of 1,5-AG was higher in the PR group compared with that in the non-PR group throughout the treatment period, although its concentration in the PR group gradually decreased. Although the serum levels of the metabolites differed significantly between the groups prior to treatment, none of the metabolites exhibited significant intergroup differences in their serum levels following treatment.

Evaluation of treatment responses: LC/MS. A total of 200, 28 and 21 metabolites were detected in the serum samples during the LC/MS-based semi-quantitative lipid, anion and cation analyses, respectively. There were a number of metabolites whose serum levels differed significantly between the PR and non-PR groups prior to the first round of therapy;
18 of these metabolites were detected in the LC/MS-based lipid analysis, and 1 metabolite, glutaconic acid, was detected in the LC/MS-based anion analysis (Table VI). By contrast, none of the metabolites detected during the LC/MS-based cation analysis exhibited significant pretreatment intergroup differences in their serum levels. A quantitative analysis of the serum levels of the 18 metabolites detected in the LC/MS-based lipid analysis or the metabolite (glutaconic acid) detected in the LC/MS-based anion analysis could not be performed, as it was difficult to obtain corresponding stable isotopes. The association between the therapeutic response and the serum levels of lipid species was examined. The resultant volcano plot of all of the lipid species detected in the present study indicated that the serum levels of fatty acids tended to be downregulated following treatment (Fig. 4), although the serum levels of a number of the other aforementioned lipid metabolites did not change significantly. This tendency was particularly pronounced for unsaturated fatty acids, polyunsaturated fatty acids (PUFAs) and monounsaturated fatty acids (Table VII). In the LC/MS-based lipid analysis conducted in the present study, free PUFAs were detected in the sera, and the serum levels of unsaturated fatty acids, in particular PUFAs, were higher in the non-PR group compared with those in the PR group. In fact, greater therapeutic effects tended to be observed in the patients with lower serum PUFA levels.

\section{Discussion}

Using metabolomics, the present study aimed to identify serum biomarkers that could be used to predict the therapeutic efficacy of chemotherapy for CRC during the pretreatment period. Metabolomics is a useful technique for identifying metabolites that are directly linked to certain molecular 
Table V. Concentrations of the targeted metabolites according to quantitative analysis.

A, Octanoic acid

\begin{tabular}{|c|c|c|c|c|}
\hline Groups & 1 st round ${ }^{\mathrm{a}}$ & 2nd round & 3rd round & 4th round \\
\hline PR & $4.09(1.00)$ & $4.79(1.03)$ & $4.44(0.96)$ & $5.27(1.14)$ \\
\hline Non-PR & $5.06(1.00)$ & $5.23(1.03)$ & $5.22(1.03)$ & $4.92(0.97)$ \\
\hline P-value ${ }^{b}$ & 0.043 & 0.391 & 0.189 & 0.901 \\
\hline
\end{tabular}

B, 1,5-AG

\begin{tabular}{lcccc}
\hline Groups & 1st round & 2nd round & 3rd round & 4th round \\
\hline PR & $155.78(1.00)$ & $145.09(0.93)$ & $116.24(0.75)$ & $117.05(0.75)$ \\
Non-PR & $106.78(1.00)$ & $106.44(1.00)$ & $108.81(1.02)$ & $101.58(0.95)$ \\
P-value $^{b}$ & 0.026 & 0.084 & 0.678 & 0.469
\end{tabular}

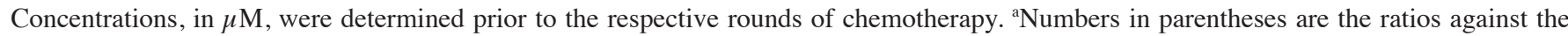
concentrations of the 1st round in each group. ${ }^{\text {b }}$-values between PR and non-PR groups were calculated using the Mann-Whitney U-test. PR, partial response; 1,5-AG, 1,5-anhydro-D-glucitol.

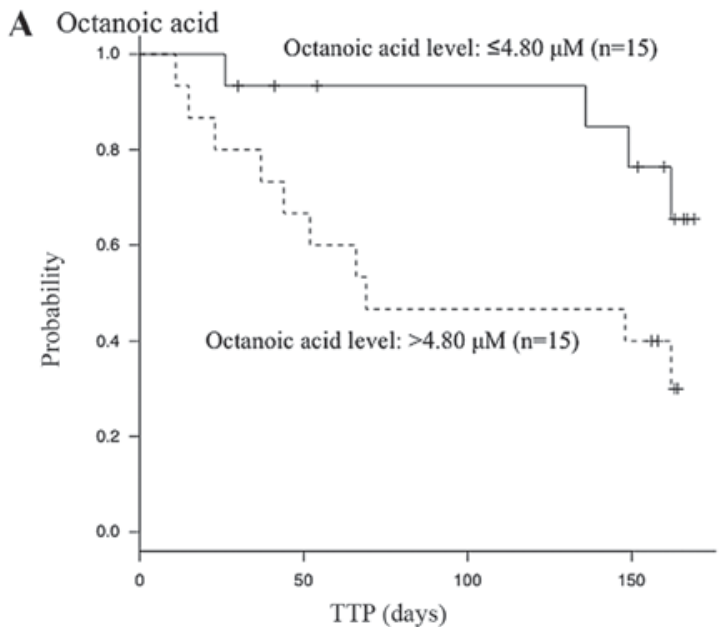

B $1,5-\mathrm{AG}$

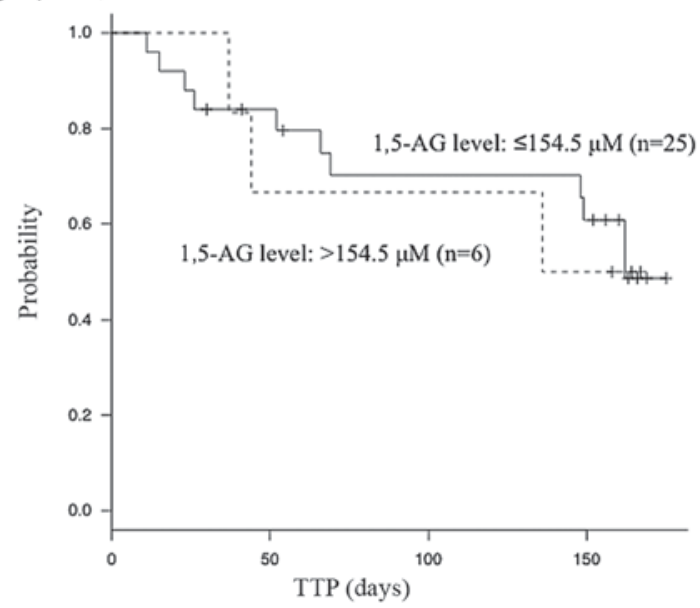

Figure 3. Kaplan-Meier curves of TTP based on the serum concentrations of the targeted metabolites. (A) Patients with lower serum octanoic acid levels, $\leq 4.80 \mu \mathrm{M}$, exhibited longer TTP compared with patients with serum octanoic acid levels of $>4.80 \mu \mathrm{M}$. (B) The analysis of 1,5-AG did not detect a significant intergroup difference. 1,5-AG, 1,5-anhydro-D-glucitol; TTP, time to progression. phenotypes. Therefore, the serum levels of metabolites were analyzed using GC/MS and LC/MS. In the semi-quantitative analysis, two metabolites, octanoic acid and 1,5-AG, were identified as biomarker candidates that could be useful for predicting the therapeutic efficacy of chemotherapy for CRC. In the quantitative analysis using the corresponding stable isotopes, it was indicated that the pretreatment serum levels of these metabolites differed significantly between the PR and non-PR groups. However, only the serum level of octanoic acid was significantly associated with the TTP, and the patients with lower serum octanoic acid levels exhibited good prognoses.

Narayanan et al (32) indicated that caprylic acid had inhibitory effects on the viability of colorectal, skin and breast cancer cells in vitro. In addition, the study reported that caprylic acid downregulated the expression of genes that are important for cell cycle division and progression in colon cancer cells, including cyclin-dependent kinase 2 (CDK2), CDK4, cyclin A2 (CCNA2) and CCND1. In another study, cetuximab increased the expression levels of the CDK inhibitors p21 and p27, and decreased the expression level of cyclin D1 by arresting the cell cycle in the $G_{1} / G_{0}$ phase (33). Based on the aforementioned findings, octanoic acid may have synergistic effects when administered in combination with cetuximab. The present study demonstrated that patients with lower serum levels of octanoic acid exhibited better prognoses compared with patients with higher serum octanoic acid levels. Yamasaki et al (34) indicated that $3 \mathrm{mM}$ octanoic acid reduced bladder cancer cell proliferation, but did not inhibit cell migration and invasion. There have been numerous studies on biomarkers of CRC involving metabolomic analysis $(35,36)$; however, to the best of our knowledge, there is a limited number of studies on octanoic acid as a biomarker candidate or on the association between the levels of octanoic acid and the prognosis of CRC. Uchiyama et al (37) performed serum metabolomic analysis using capillary electrophoresis-time of-flight MS in order to identify biomarkers that may be used 
Table VI. The significantly-altered metabolites in LC/MS-based semi-quantitative lipid and anion analysis.

\begin{tabular}{|c|c|c|c|c|c|c|}
\hline \multirow[b]{2}{*}{ Metabolomics } & \multicolumn{2}{|c|}{$\mathrm{PR}(\mathrm{n}=7)$} & \multicolumn{2}{|c|}{ Non-PR $(n=24)$} & \multirow[b]{2}{*}{ Fold-change $^{a}$} & \multirow[b]{2}{*}{ P-value ${ }^{b}$} \\
\hline & Mean & SD & Mean & $\mathrm{SD}$ & & \\
\hline \multicolumn{7}{|l|}{ Lipid analysis } \\
\hline \multicolumn{7}{|l|}{ Fatty acids } \\
\hline $\begin{array}{l}\text { 18:1 (n-9)_trans-elaidic acid } \\
\text { and (n-7)_trans-vaccenic acid }\end{array}$ & 0.1611 & 0.0354 & 0.1334 & 0.0991 & 1.208 & 0.042 \\
\hline 18:2(n-6)_linoleic acid & 39.2849 & 3.5305 & 44.5618 & 6.6877 & 0.882 & 0.047 \\
\hline 20:2 (n-6)_cis-11-14-eicosadienoic acid & 0.8759 & 0.4409 & 1.8429 & 1.3528 & 0.475 & 0.026 \\
\hline $20: 4$ (n-6)_arachidonic acid & 0.0097 & 0.0031 & 0.0271 & 0.0206 & 0.356 & 0.021 \\
\hline 22:4 (n-6)_docosatetraenoic acid & 0.0331 & 0.0106 & 0.0846 & 0.0647 & 0.391 & 0.003 \\
\hline 20:1 (n-9)_cis-11-eicosenoic acid & 3.1298 & 1.4758 & 6.8503 & 5.5053 & 0.457 & 0.016 \\
\hline 24:1 (n-9)_nervonic acid & 0.1312 & 0.0615 & 0.2411 & 0.1377 & 0.544 & 0.034 \\
\hline 26:0_cerotic acid & 0.0815 & 0.0198 & 0.1228 & 0.0408 & 0.663 & 0.034 \\
\hline \multicolumn{7}{|l|}{ Phosphatidylcholines } \\
\hline PC (16:0e/18:2) & 0.0074 & 0.0058 & 0.0159 & 0.0112 & 0.465 & 0.023 \\
\hline PC (16:0/20:4) \& PC (16:1/20:3) & 7.1136 & 0.9062 & 6.4849 & 2.4726 & 1.097 & 0.023 \\
\hline PC (17:0/16:0) \& PC (18:0/15:0) & 3.6935 & 1.0740 & 2.6142 & 0.9398 & 1.413 & 0.030 \\
\hline PC (18:0/20:4) & 0.6628 & 0.0808 & 0.5768 & 0.2268 & 1.149 & 0.042 \\
\hline PC (18:1e/18:2) & 0.2680 & 0.0849 & 0.3612 & 0.1006 & 0.742 & 0.026 \\
\hline PC (18:1e/16:0) \& PC (18:0e/16:1) & 2.8203 & 0.7449 & 2.1057 & 0.7165 & 1.339 & 0.042 \\
\hline \multicolumn{7}{|l|}{ Phosphatidylethanolamines } \\
\hline PE (16:0/20:4) & 0.1779 & 0.0691 & 0.2674 & 0.0946 & 0.665 & 0.005 \\
\hline PE (18:0/20:4) & 0.1160 & 0.0456 & 0.3105 & 0.2602 & 0.374 & 0.034 \\
\hline PE (20:0/18:2) & 51.0289 & 5.6977 & 58.8697 & 8.3221 & 0.867 & 0.030 \\
\hline PE (20:1/18:2) & 0.0185 & 0.0041 & 0.0327 & 0.0155 & 0.567 & 0.014 \\
\hline \multicolumn{7}{|l|}{ Anion analysis } \\
\hline Glutaconic acid & 0.1467 & 0.0618 & 0.0941 & 0.0460 & 1.559 & 0.047 \\
\hline
\end{tabular}

${ }^{a}$ The fold-change values were calculated based on the ratio of PR to non-PR. ${ }^{b}$ The P-values between PR and non-PR groups were calculated using the Mann-Whitney U-test. PR, partial response; Non-PR, non-partial response; SD, standard deviation; FA, fatty acids; PC, phosphatidylcholines; PE, phosphatidylethanolamines.

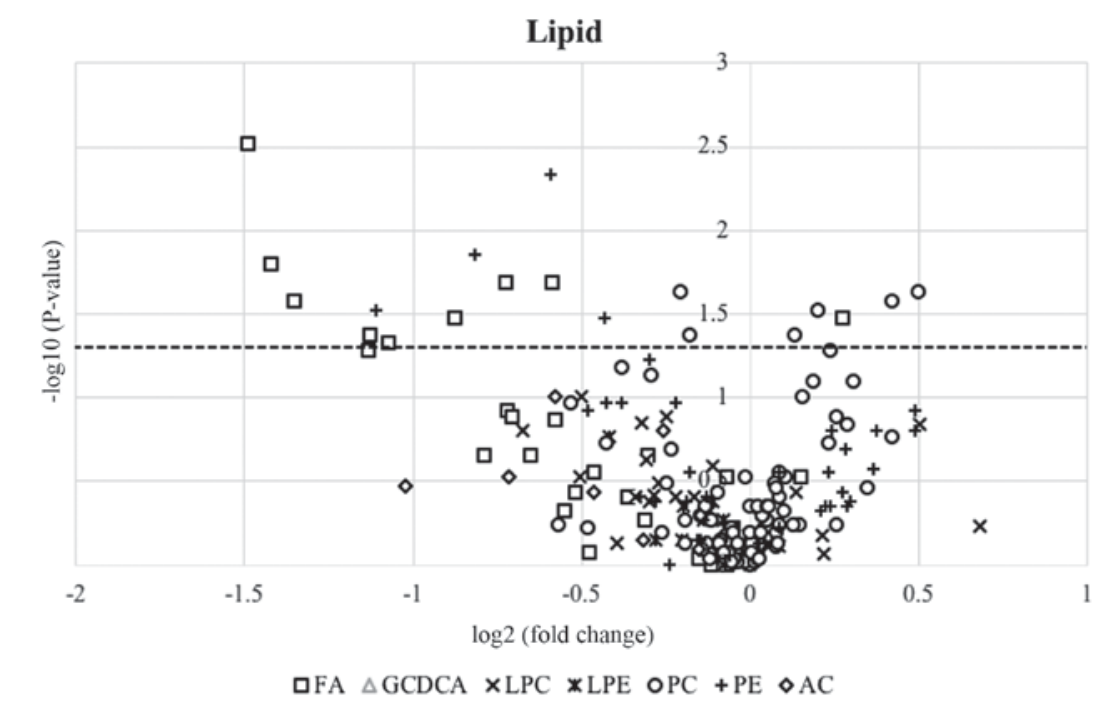

Figure 4. Volcano plot analysis of the lipid species. X axis, the PR to non-PR ratio on a $\log 2$ scale; Y axis, -log10 (P-value). The P-values were calculated using the Mann-Whitney U-test and the horizontal dashed line represents a P-value of 0.05. AC, acylcarnitines; FA, fatty acids; GCDCA, glycochenodeoxycholic acid; LPC lysophosphatidylcholines; LPE, lysophosphatidylethanolamines; PC, phosphatidylcholines; PE, phosphatidylethanolamines; PR, partial response. 
Table VII. Detailed results of the LC/MS-based semi-quantitative analysis of serum fatty acids.

\begin{tabular}{|c|c|c|c|c|c|c|}
\hline \multirow[b]{2}{*}{ Serum fatty acids } & \multicolumn{2}{|c|}{$\mathrm{PR}(\mathrm{n}=7)$} & \multicolumn{2}{|c|}{ Non-PR $(n=24)$} & \multirow[b]{2}{*}{ Fold-change $^{\mathrm{a}}$} & \multirow[b]{2}{*}{ P-value ${ }^{b}$} \\
\hline & Mean & $\mathrm{SD}$ & Mean & SD & & \\
\hline \multicolumn{7}{|l|}{ Saturated fatty acids } \\
\hline 12:0_lauric acid & 0.085 & 0.035 & 0.088 & 0.037 & 0.97 & 0.60 \\
\hline 14:0_myristic acid & 0.223 & 0.084 & 0.277 & 0.148 & 0.81 & 0.54 \\
\hline 15:0_pentadecylic acid & 0.024 & 0.010 & 0.034 & 0.023 & 0.70 & 0.37 \\
\hline 16:0_palmitic acid & 3.736 & 1.627 & 5.473 & 4.126 & 0.68 & 0.48 \\
\hline 17:0_margaric acid & 0.047 & 0.015 & 0.052 & 0.031 & 0.90 & 0.92 \\
\hline 18:0_stearic acid & 3.294 & 0.816 & 4.238 & 2.306 & 0.78 & 0.39 \\
\hline 20:0_arachidic acid & 0.076 & 0.026 & 0.080 & 0.033 & 0.95 & 1.00 \\
\hline 21:0_heneicosanoic acid & 0.026 & 0.015 & 0.027 & 0.012 & 0.96 & 0.92 \\
\hline 22:0_behenic acid & 0.098 & 0.020 & 0.106 & 0.050 & 0.92 & 1.00 \\
\hline 23:0_tricosanoic acid & 0.061 & 0.023 & 0.055 & 0.038 & 1.11 & 0.30 \\
\hline 24:0_lignoceric acid & 0.209 & 0.037 & 0.219 & 0.140 & 0.95 & 0.30 \\
\hline 25:0_pentacosanoic acid & 0.072 & 0.048 & 0.100 & 0.182 & 0.72 & 0.85 \\
\hline 26:0_cerotic acid & 0.161 & 0.035 & 0.133 & 0.099 & 1.21 & 0.03 \\
\hline 27:0_heptacosanoic acid & 0.024 & 0.016 & 0.024 & 0.019 & 0.98 & 0.92 \\
\hline \multicolumn{7}{|l|}{ Polyunsaturated fatty acids } \\
\hline 18:2(n-6)_linoleic acid & 0.876 & 0.441 & 1.843 & 1.353 & 0.48 & 0.05 \\
\hline 20:2 (n-6)_cis-11-14-eicosadienoic acid & 0.033 & 0.011 & 0.085 & 0.065 & 0.39 & 0.03 \\
\hline $\begin{array}{l}\text { 20:3 (n-6)_dihomo- } \gamma \text {-linolenic acid } \\
\text { and (n-9)_mead acid }\end{array}$ & 0.036 & 0.016 & 0.054 & 0.030 & 0.67 & 0.13 \\
\hline 20:4 (n-6)_arachidonic acid & 0.178 & 0.069 & 0.267 & 0.095 & 0.67 & 0.02 \\
\hline 22:4 (n-6)_docosatetraenoic acid & 0.010 & 0.003 & 0.027 & 0.021 & 0.36 & 0.00 \\
\hline 22:5(n-6)_docosapentaenoic acid & 0.099 & 0.074 & 0.162 & 0.128 & 0.61 & 0.13 \\
\hline $\begin{array}{l}\text { 18:3 }(n-3) \_\alpha \text {-linolenic acid } \\
\text { and }(n-6) \_\gamma \text {-linolenic acid }\end{array}$ & 0.125 & 0.093 & 0.173 & 0.096 & 0.73 & 0.28 \\
\hline 20:5 (n-3)_eicosapentaenoic acid & 0.051 & 0.033 & 0.063 & 0.027 & 0.81 & 0.22 \\
\hline 22:6(n-3)_docosahexaenoic acid & 0.122 & 0.066 & 0.203 & 0.093 & 0.60 & 0.02 \\
\hline \multicolumn{7}{|l|}{ Monounsaturated fatty acids } \\
\hline 14:1(n-5)_myristoleic acid & 0.032 & 0.020 & 0.054 & 0.036 & 0.61 & 0.12 \\
\hline 16:1 (n-7)_palmitoleic acid & 0.344 & 0.171 & 0.595 & 0.466 & 0.58 & 0.22 \\
\hline 17:1 (n-7)_cis-10-heptadecanoic acid & 0.085 & 0.045 & 0.134 & 0.096 & 0.64 & 0.22 \\
\hline $\begin{array}{l}\text { 18:1 (n-9)_cis-oleic acid } \\
\text { and (n-7)_cis-vaccenic acid }\end{array}$ & 1.305 & 0.603 & 2.867 & 2.314 & 0.46 & 0.05 \\
\hline $\begin{array}{l}\text { 18:1 (n-9)_trans-elaidic acid } \\
\text { and (n-7)_trans-vaccenic acid }\end{array}$ & 3.130 & 1.476 & 6.850 & 5.505 & 0.46 & 0.04 \\
\hline 20:1 (n-9)_cis-11-eicosenoic acid & 0.116 & 0.046 & 0.311 & 0.260 & 0.37 & 0.02 \\
\hline 24:1 (n-9)_nervonic acid & 0.131 & 0.061 & 0.241 & 0.138 & 0.54 & 0.03 \\
\hline
\end{tabular}

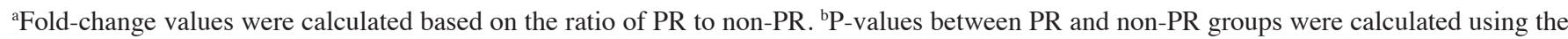
Mann-Whitney U-test. PR, partial response; SD, standard deviation.

for the early detection of CRC. As a result, it was indicated that the serum level of octanoic acid increased with the clinical stage of $\mathrm{CRC}$, but the diagnostic power of octanoic acid alone for detecting cancer did not suffice. Conversely, it was demonstrated that the serum level of octanoic acid was higher in patients with colorectal adenoma compared with that in the controls, so octanoic acid may be a useful biomarker for diagnosing adenoma. Octanoic acid is a dietary metabolite. Jansen et al (38) reported that the intake of full fat products, which contain saturated fatty acids, including octanoic acid, increases the risk of pancreatic cancer in a dose-dependent manner. However, to the best of our knowledge, no studies examining the influence of full fat products or octanoic acid on the risk of $\mathrm{CRC}$ have been reported, and therefore, further investigation is required.

$1,5-\mathrm{AG}$ is a non-metabolizable glucose analogue that is found in plasma (39). The plasma 1,5-AG level has been proposed as a marker of glycemic control in patients with 
diabetes. In addition, hyperinsulinemia and insulin resistance have been reported as risk factors for cancer, including CRC (40). In the present study, the patients with lower serum levels of 1,5-AG exhibited worse prognoses compared with the patients with higher serum 1,5-AG levels. It is unlikely that this result was affected by the frequency of pretreatment diabetes, as only 1 of the 31 patients had a medical history of diabetes. By contrast, Meyerhardt et al (41) suggested that a higher dietary glycemic load and a greater total carbohydrate intake had significant associations with an increased risk of recurrence and mortality in patients with stage III CRC, based on investigations of the dietary habits of patients and the calculation of glycemic index values. These results are consistent with the findings of the present study indicating that patients with lower serum levels of 1,5-AG exhibited worse prognoses. However, further studies are required in order to fully elucidate these associations.

It has been reported that $\mathrm{n}-3$ PUFAs have anti-inflammatory and anticancer effects, the latter of which involve the enhancement of oxidative stress in cancer cells $(42,43)$. In addition, n-3 PUFAs enhance the efficacy of treatment in a dose-dependent manner when combined with chemotherapy and radiotherapy $(44,45)$. By contrast, n-6 PUFAs promote colorectal carcinogenesis via inflammation and peroxidation $(46,47)$. In the LC/MS-based lipid analysis conducted in the present study, greater therapeutic effects were observed in the patients with lower serum PUFA levels. One possible explanation for these findings is that n-6 PUFAs, which are linked with carcinogenesis, make a greater contribution to therapeutic responses compared with n-3 PUFAs. Furthermore, Hardy et al (48) confirmed that the effects of free fatty acids on breast cancer (e.g., on proliferation and apoptosis) depended on the types of fatty acids and the relative frequencies of oleic acid and palmitic acid. According to the aforementioned findings, not only the concentration of each fatty acid, but also the proportions of each type of fatty acid, including saturated, monounsaturated and polyunsaturated, may influence carcinogenesis and proliferation in CRC, and hence, could affect therapeutic efficacy.

The present study presented with a number of limitations. First, the serum levels of the targeted metabolites may have been changed not only by the chemotherapy, but also by the decline in the general condition of the patients due to their underlying diseases. In the present study, the serum samples were obtained from patients with CRC for which second-line chemotherapy had failed and who therefore may have had subclinical organ disorders. Therefore, the serum levels of the targeted metabolites may have exhibited a more distinct change in the event that serum samples were used from patients who had been treated with cetuximab plus irinotecan, or cetuximab alone as a first-line chemotherapy. Secondly, it is unclear whether the aforementioned biomarkers are specific to cetuximab therapy or whether they are applicable to all chemotherapies for CRC. Thirdly, it was difficult to evaluate why the aforementioned serum metabolites appeared to be useful as biomarkers for predicting the efficacy of chemotherapy in patients with CRC due to the limited number of existing studies. Fourthly, the number of samples was low as the study was attached to a phase II trial of chemotherapy. Fifth, the diagnostic power of these metabolites was not validated using other cohorts. Therefore, a further large-scale study is required to evaluate the results of the present study.

The aim of the present study was to establish biomarkers for predicting the efficacy of chemotherapy for CRC. The serum metabolite levels were analyzed in patients with CRC who were treated with chemotherapy consisting of cetuximab plus irinotecan or cetuximab alone. In such patient cases, changes in metabolite concentrations can be induced by treatment and/or disease progression. Therefore, it may be difficult to identify such biomarkers following treatment, and therefore the study focused on serum metabolites that could be used as predictive biomarkers prior to treatment. As a result, octanoic acid and 1,5-AG were identified as biomarker candidates for predicting the efficacy of chemotherapy for CRC. In the quantitative analysis, the serum concentrations of octanoic acid and 1,5-AG differed significantly between the PR and the non-PR groups. In addition, the present study suggests that the serum concentration of octanoic acid is useful for predicting the prognosis of patients with CRC. Therefore, the findings of the present study may aid in improving the quality of life and prognosis of patients with CRC who are treated with chemotherapy.

\section{Acknowledgements}

Not applicable.

\section{Funding}

The present study was supported in part by a Grant-in-Aid for Scientific Research from the Japan Society for the Promotion of Science (grant no. 16H05227) and the AMED-CREST program of the Japan Agency for Medical Research and Development (grant no. 17gm0710013h0004).

\section{Availability of data and materials}

The datasets used and/or analyzed during the current study are available from the corresponding author on reasonable request.

\section{Authors' contributions}

SN, TK, TH, KK, HS, YM, KH and MY conceived the idea for the present study, designed the study and assisted in drafting the manuscript. TI and SF analyzed and interpreted the data. $\mathrm{TH}, \mathrm{KK}, \mathrm{HS}, \mathrm{YM}$ and $\mathrm{KH}$ collected the specimens and patient information. TI wrote the manuscript. All of the authors read and approved the final manuscript.

\section{Ethics approval and consent to participate}

The present study was approved by the Ethics Committees of the National Cancer Center Hospital (Tokyo, Japan; approval no. G2008-005) and Kobe University Graduate School of Medicine (Kobe, Japan; approval no. 1833) and was performed in accordance to the principles outlined in the Declaration of Helsinki for all human and animal experiments. In addition, in the investigations involving human subjects, written informed consent was obtained from all subjects. 


\section{Patient consent for publication}

Informed consent for the publication of any data/ associated images was obtained in from all participants.

\section{Competing interests}

The authors declare that they have no competing interests.

\section{References}

1. Watanabe T, Muro K, Ajioka Y, Hashiguchi Y, Ito Y, Saito Y, Hamaguchi $\mathrm{T}$, Ishida $\mathrm{H}$, Ishiguro $\mathrm{M}$, Ishihara $\mathrm{S}$, et al; Japanese Society for Cancer of the Colon and Rectum: Japanese Society for Cancer of the Colon and Rectum (JSCCR) guidelines 2016 for the treatment of colorectal cancer. Int J Clin Oncol 23: 1-34, 2018.

2. Moertel CG: Chemotherapy for colorectal cancer. N Engl J Med 330: 1136-1142, 1994.

3. Saltz LB, Cox JV, Blanke C, Rosen LS, Fehrenbacher L, Moore MJ, Maroun JA, Ackland SP, Locker PK, Pirotta N, et al; Irinotecan Study Group: Irinotecan plus fluorouracil and leucovorin for metastatic colorectal cancer. N Engl J Med 343: 905-914, 2000.

4. Colucci G, Gebbia V, Paoletti G, Giuliani F, Caruso M, Gebbia N, Cartenì G, Agostara B, Pezzella G, Manzione L, et al; Gruppo Oncologico Dell'Italia Meridionale: Phase III randomized trial of FOLFIRI versus FOLFOX4 in the treatment of advanced colorectal cancer: A multicenter study of the Gruppo Oncologico Dell'Italia Meridionale. J Clin Oncol 23: 4866-4875, 2005.

5. Goldberg RM, Sargent DJ, Morton RF, Fuchs CS, Ramanathan RK, Williamson SK, Findlay BP, Pitot HC and Alberts SR: A randomized controlled trial of fluorouracil plus leucovorin, irinotecan, and oxaliplatin combinations in patients with previously untreated metastatic colorectal cancer. J Clin Oncol 22: 23-30, 2004.

6. Hurwitz H, Fehrenbacher L, Novotny W, Cartwright T, Hainsworth J, Heim W, Berlin J, Baron A, Griffing S, Holmgren E, et al: Bevacizumab plus irinotecan, fluorouracil, and leucovorin for metastatic colorectal cancer. N Engl J Med 350: 2335-2342, 2004.

7. Hochster HS, Hart LL, Ramanathan RK, Childs BH, Hainsworth JD, Cohn AL, Wong L, Fehrenbacher L, Abubakr Y, Saif MW, et al: Safety and efficacy of oxaliplatin and fluoropyrimidine regimens with or without bevacizumab as first-line treatment of metastatic colorectal cancer: Results of the TREE Study. J Clin Oncol 26: 3523-3529, 2008.

8. Jonker DJ, O'Callaghan CJ, Karapetis CS, Zalcberg JR, Tu D, Au HJ, Berry SR, Krahn M, Price T, Simes RJ, et al: Cetuximab for the treatment of colorectal cancer. N Engl J Med 357: 2040-2048, 2007.

9. Cunningham D, Humblet $\mathrm{Y}$, Siena S, Khayat D, Bleiberg H, Santoro A, Bets D, Mueser M, Harstrick A, Verslype C, et al: Cetuximab monotherapy and cetuximab plus irinotecan in irinotecan-refractory metastatic colorectal cancer. N Engl J Med 351: 337-345, 2004.

10. De Roock W, Piessevaux H, De Schutter J, Janssens M, De Hertogh G, Personeni N, Biesmans B, Van Laethem JL, Peeters M, Humblet Y, et al: KRAS wild-type state predicts survival and is associated to early radiological response in metastatic colorectal cancer treated with cetuximab. Ann Oncol 19: 508-515, 2008

11. Lièvre A, Bachet JB, Le Corre D, Boige V, Landi B, Emile JF Côté JF, Tomasic G, Penna C, Ducreux M, et al: KRAS mutation status is predictive of response to cetuximab therapy in colorectal cancer. Cancer Res 66: 3992-3995, 2006.

12. Di Nicolantonio F, Martini M, Molinari F, Sartore-Bianchi A, Arena S, Saletti P, De Dosso S, Mazzucchelli L, Frattini M, Siena $\mathrm{S}$, et al: Wild-type BRAF is required for response to panitumumab or cetuximab in metastatic colorectal cancer. J Clin Oncol 26: 5705-5712, 2008.

13. Kasper S, Reis H, Ziegler S, Nothdurft S, Mueller A, Goetz M, Wiesweg M, Phasue J, Ting S, Wieczorek S, et al: Molecular dissection of effector mechanisms of RAS-mediated resistance to anti-EGFR antibody therapy. Oncotarget 8: 45898-45917, 2017.
14. Van Cutsem E, Köhne CH, Láng I, Folprecht G, Nowacki MP, Cascinu S, Shchepotin I, Maurel J, Cunningham D, Tejpar S, et al: Cetuximab plus irinotecan, fluorouracil, and leucovorin as first-line treatment for metastatic colorectal cancer: Updated analysis of overall survival according to tumor KRAS and BRAF mutation status. J Clin Oncol 29: 2011-2019, 2011.

15. Yoshida M, Hatano N, Nishiumi S, Irino Y, Izumi Y, Takenawa T and Azuma T: Diagnosis of gastroenterological diseases by metabolome analysis using gas chromatography-mass spectrometry. J Gastroenterol 47: 9-20, 2012.

16. Spratlin JL, Serkova NJ and Eckhardt SG: Clinical applications of metabolomics in oncology: a review. Clin Cancer Res 15: 431-440, 2009.

17. Fernández-Ochoa Á, Quirantes-Piné R, Borrás-Linares I Gemperline D, Alarcón Riquelme ME, Beretta L and Segura-Carretero A; PRECISESADS Clinical Consortium: Urinary and plasma metabolite differences detected by HPLC-ESI-QTOF-MS in systemic sclerosis patients. J Pharm Biomed Anal 162: 82-90, 2018.

18. Jeong A, Fiorito G, Keski-Rahkonen P, Imboden M, Kiss A, Robinot N, Gmuender H, Vlaanderen J, Vermeulen R, Kyrtopoulos S, et al; EXPOsOMICS Consortium: Perturbation of metabolic pathways mediates the association of air pollutants with asthma and cardiovascular diseases. Environ Int 119: 334-345, 2018.

19. Kobayashi T, Nishiumi S, Ikeda A, Yoshie T, Sakai A, Matsubara A, Izumi Y, Tsumura H, Tsuda M, Nishisaki $\mathrm{H}$, et al: A novel serum metabolomics-based diagnostic approach to pancreatic cancer. Cancer Epidemiol Biomarkers Prev 22: 571-579, 2013.

20. Sakai A, Suzuki M, Kobayashi T, Nishiumi S, Yamanaka K, Hirata Y, Nakagawa T, Azuma T and Yoshida M: Pancreatic cancer screening using a multiplatform human serum metabolomics system. Biomark Med 10: 577-586, 2016.

21. Hirata Y, Kobayashi T, Nishiumi S, Yamanaka K, Nakagawa T, Fujigaki S, Iemoto T, Kobayashi M, Okusaka T, Nakamori S, et al: Identification of highly sensitive biomarkers that can aid the early detection of pancreatic cancer using GC/MS/MS-based targeted metabolomics. Clin Chim Acta 468: 98-104, 2017.

22. Nishiumi S, Kobayashi T, Kawana S, Unno Y, Sakai T, Okamoto K, Yamada Y, Sudo K, Yamaji T, Saito Y, et al: Investigations in the possibility of early detection of colorectal cancer by gas chromatography/triple-quadrupole mass spectrometry. Oncotarget 8: 17115-17126, 2017.

23. Redalen KR, Sitter B, Bathen TF, Grøholt KK, Hole KH, Dueland S, Flatmark K, Ree AH and Seierstad T: High tumor glycine concentration is an adverse prognostic factor in locally advanced rectal cancer. Radiother Oncol 118: 393-398, 2016.

24. Terazawa T, Nishitani H, Kato K, Hashimoto H, Akiyoshi K, Ito Y, Nakamoto A, Iwasa S, Nakajima TE, Hamaguchi T, et al: Phase II study of cetuximab with irinotecan for KRAS wild-type colorectal cancer in Japanese patients. Asia Pac J Clin Oncol 13: e132-e137, 2017.

25. Oken MM, Creech RH, Tormey DC, Horton J, Davis TE, McFadden ET and Carbone PP: Toxicity and response criteria of the Eastern Cooperative Oncology Group. Am J Clin Oncol 5: 649-655, 1982.

26. Therasse P, Arbuck SG, Eisenhauer EA, Wanders J, Kaplan RS, Rubinstein L, Verweij J, Van Glabbeke M, van Oosterom AT, Christian MC, and Gwyther SG: New guidelines to evaluate the response to treatment in solid tumors. European Organization for Research and Treatment of Cancer, National Cancer Institute of the United States, National Cancer Institute of Canada. J Natl Cancer Inst 92: 205-216, 2000.

27. Fujigaki S, Nishiumi S, Kobayashi T, Suzuki M, Iemoto T, Kojima T, Ito Y, Daiko H, Kato K, Shouji H, et al: Identification of serum biomarkers of chemoradiosensitivity in esophageal cancer via the targeted metabolomics approach. Biomarkers Med 12: 827-840, 2018

28. Yamashita Y, Nishiumi S, Kono S, Takao S, Azuma T and Yoshida M: Differences in elongation of very long chain fatty acids and fatty acid metabolism between triple-negative and hormone receptor-positive breast cancer. BMC Cancer 17: 589, 2017.

29. Youden WJ: Index for rating diagnostic tests. Cancer 3: 32-35, 1950.

30. Kanda Y: Investigation of the freely available easy-to-use software 'EZR' for medical statistics. Bone Marrow Transplant 48: 452-458, 2013 
31. Birkemeyer C, Luedemann A, Wagner C, Erban A and Kopka J: Metabolome analysis: The potential of in vivo labeling with stable isotopes for metabolite profiling. Trends Biotechnol 23: $28-33,2005$.

32. Narayanan A, Baskaran SA, Amalaradjou MAR and Venkitanarayanan K: Anticarcinogenic properties of medium chain fatty acids on human colorectal, skin and breast cancer cells in vitro. Int J Mol Sci 16: 5014-5027, 2015.

33. Huether A, Höpfner M, Baradari V, Schuppan D and Scherübl H: EGFR blockade by cetuximab alone or as combination therapy for growth control of hepatocellular cancer. Biochem Pharmacol 70: 1568-1578, 2005.

34. Yamasaki M, Soda S, Sakakibara Y, Suiko M and Nishiyama K: The importance of 1,2-dithiolane structure in $\alpha$-lipoic acid for the downregulation of cell surface $\beta 1$-integrin expression of human bladder cancer cells. Biosci Biotechnol Biochem 78: 1939-1942, 2014

35. Djukovic D, Zhang J and Raftery D: Colorectal cancer detection using targeted LC-MS metabolic profiling. Methods Mol Biol 1765: 229-240, 2018.

36. Delphan M, Lin T, Liesenfeld DB, Nattenmüller J, Böhm JT, Gigic B, Habermann N, Zielske L, Schrotz-King P, Schneider M, et al: Associations of branched-chain amino acids with parameters of energy balance and survival in colorectal cancer patients: Results from the ColoCare Study. Metabolomics 2018: 22, 2018.

37. Uchiyama K, Yagi N, Mizushima K, Higashimura Y, Hirai Y, Okayama T, Yoshida N, Katada K, Kamada K, Handa O, et al: Serum metabolomics analysis for early detection of colorectal cancer. J Gastroenterol 52: 677-694, 2017.

38. Jansen RJ, Robinson DP, Frank RD, Anderson KE, Bamlet WR, Oberg AL, Rabe KG, Olson JE, Sinha R, Petersen GM, et al: Fatty acids found in dairy, protein and unsaturated fatty acids are associated with risk of pancreatic cancer in a case-control study. Int J Cancer 134: 1935-1946, 2014.

39. Sato T, Kameyama T and Inoue H: Association of reduced levels of serum 1,5-Anhydro-d-glucitol with carotid atherosclerosis in patients with type 2 diabetes. J Diabetes Complications 28 : 348-352, 2014

40. Wang X, Häring M-F, Rathjen T, Lockhart SM, Sørensen D, Ussar S, Rasmussen LM, Bertagnolli MM, Kahn CR and Rask-Madsen C: Insulin resistance in vascular endothelial cells promotes intestinal tumour formation. Oncogene 36: 4987-4996, 2017.
41. Meyerhardt JA, Sato K, Niedzwiecki D, Ye C, Saltz LB, Mayer RJ, Mowat RB, Whittom R, Hantel A, Benson A, et al: Dietary glycemic load and cancer recurrence and survival in patients with stage III colon cancer: Findings from CALGB 89803. J Natl Cancer Inst 104: 1702-1711, 2012.

42. Larsson SC, Kumlin M, Ingelman-Sundberg $M$ and Wolk A: Dietary long-chain n-3 fatty acids for the prevention of cancer: A review of potential mechanisms. Am J Clin Nutr 79: 935-945, 2004.

43. Hall MN, Chavarro JE, Lee IM, Willett WC and Ma J: A 22-year prospective study of fish, n-3 fatty acid intake, and colorectal cancer risk in men. Cancer Epidemiol Biomarkers Prev 17: 1136-1143, 2008

44. Siena L, Cipollina C, Di Vincenzo S, Ferraro M, Bruno A, Gjomarkaj M and Pace E: Electrophilic derivatives of omega-3 fatty acids counteract lung cancer cell growth. Cancer Chemother Pharmacol 81: 705-716, 2018.

45. Benais-Pont G, Dupertuis YM, Kossovsky MP, Nouet P, Allal AS, Buchegger F and Pichard C: Omega-3 polyunsaturated fatty acids and ionizing radiation: Combined cytotoxicity on human colorectal adenocarcinoma cells. Nutrition 22: 931-939, 2006.

46. Cai F, Dupertuis YM and Pichard C: Role of polyunsaturated fatty acids and lipid peroxidation on colorectal cancer risk and treatments. Curr Opin Clin Nutr Metab Care 15: 99-106, 2012.

47. Yang K, Li H, Dong J, Dong Y and Wang CZ: Expression profile of polyunsaturated fatty acids in colorectal cancer. World J Gastroenterol 21: 2405-2412, 2015

48. Hardy S, Langelier Y and Prentki M: Oleate activates phosphatidylinositol 3-kinase and promotes proliferation and reduces apoptosis of MDA-MB-231 breast cancer cells, whereas palmitate has opposite effects. Cancer Res 60: 6353-6358, 2000 . 